\title{
High-energy pseudogap induced by Hund coupling in a degenerate Hubbard model
}

\author{
Ansgar Liebsch \\ Peter Grünberg Institute and Institute of Advanced Simulations, Forschungszentrum Jülich, D-52425 Jülich, Germany
}

(Received 15 August 2011; revised manuscript received 20 September 2011; published 11 November 2011)

\begin{abstract}
Hund coupling in the degenerate five-band Hubbard model near $n=6$ occupancy is shown to give rise to a significant depletion of spectral weight above the Fermi level. Calculations within dynamical mean-field theory combined with exact diagonalization reveal that this pseudogap is associated with a collective mode in the self-energy caused by spin fluctuations. The pseudogap is remarkably stable over a wide range of Coulomb and exchange energies, but disappears for weak Hund coupling. The implications of this phenomenon for optical spectra of iron pnictides are discussed.
\end{abstract}

DOI: 10.1103/PhysRevB.84.180505

PACS number(s): 74.70.Xa, 71.27.+a

Introduction. The discovery of superconductivity in iron pnictides ${ }^{1}$ has stimulated intense discussions concerning the role of correlation effects in these compounds. In contrast to high- $T_{c}$ cuprates, which have antiferromagnetic Mott insulators as parent compounds, pnictides are correlated magnetic metals that tend to show significant deviations from Fermi-liquid behavior. Moreover, as a result of the multiband nature of pnictides, the interplay of Coulomb and exchange interactions gives rise to phenomena not found in cuprates. The importance of Hund coupling in pnictides and chalcogenides was recently pointed out in several theoretical ${ }^{2-6}$ and experimental studies. ${ }^{7-9}$ Optical data on paramagnetic LaFePO (Ref. 10) and $\mathrm{BaFe}_{2} \mathrm{As}_{2}$ (Refs. 7-9) reveal a high-energy pseudogap not compatible with normal metal behavior. This pseudogap differs fundamentally from the low-energy gap in the antiferromagnetic spin-density wave phase. Also, angleresolved photoemission spectra for doped $\mathrm{BaFe}_{2} \mathrm{As}_{2}$ exhibit a depletion of spectral weight near the Fermi level that differs from the superconducting gap. ${ }^{11}$

To investigate the influence of Coulomb correlations on the electronic properties of iron pnictides, various groups ${ }^{2,6,12-21}$ have used dynamical mean-field theory ${ }^{22}$ (DMFT) combined with single-particle Hamiltonians derived from densityfunctional theory. These calculations typically revealed moderate to strong effective mass enhancement, in agreement with experimental data. Correlations were also shown to give rise to nonzero low-energy scattering rates, ${ }^{13,16,18}$ indicating bad metallicity. On the other hand, as a result of the complex band structure of pnictides, all five $d$ bands are important, so that the many-body features exhibit a marked orbital dependence. Moreover, the $\mathrm{Fe} 3 d$ density of states generally shows two main features separated by a deep minimum slightly above the Fermi energy. In view of these multiband characteristics, it is difficult to distinguish genuine many-body features from single-particle properties. For instance, it is presently not clear to what extent pseudogaps in the interacting density of states are induced by Coulomb correlations or band-structure effects.

The aim of the present Rapid Communication is to unravel these competing influences. For this purpose we have performed DMFT calculations for a simplified Hamiltonian consisting of five degenerate semielliptical bands. Since the density of states is featureless, correlation-induced spectral modifications are easily identified. In addition, by scanning a wide range of Coulomb and exchange energies, it is feasible to investigate their respective roles. The main result of this Rapid Communication is that many-body effects are much more sensitive to the magnitude of Hund's rule coupling $J$ than to the intraorbital Coulomb repulsion $U$. In particular, exchange interactions give rise to a pseudogap above $E_{F}$, which persists in the full range of realistic values of $J$, but disappears at small $J$. Within the present model, this pseudogap can be linked to a resonance in the self-energy caused by spin fluctuations. We also argue that these correlation effects can be understood qualitatively by viewing the system at $n=6$ occupancy as a doped $n=5$ Mott insulator. Because of the high orbital degeneracy, the paramagnetic half-filled system is metallic at small $J$, but insulating already at moderate Hund coupling. Upon electron doping, a narrow quasiparticle peak appears in the density of states, with a maximum below and a pseudogap above $E_{F}$. Thus, as a function of $J$, the pseudogap at $n=6$ exists only in proximity to the Mott phase of the half-filled system.

Results and discussion. To motivate the present work, we show first in Fig. 1 a comparison of the electron spectral distribution of FeAsLaO derived within DMFT with the bare density of states obtained from the effective $3 d$ single-particle Hamiltonian provided in Ref. 23. Both spectra exhibit a striking minimum slightly above $E_{F}$. As an impurity solver we use exact diagonalization (ED). ${ }^{24}$ Each $d$ orbital hybridizes with one or two bath levels, giving total cluster sizes $n_{s}=10$ or $n_{s}=15$, respectively. The interacting Hamiltonian and additional details concerning the ED DMFT approach are specified in Ref. 16. Coulomb and exchange interactions are treated in a rotationally invariant manner. The temperature of the Matsubara grid corresponds to $T \approx 0.01 \mathrm{eV}$, while the interacting cluster Green's function is evaluated at $T=0$ for computational reasons. The spectral distribution at real $\omega$ is obtained by using the routine ratint (Ref. 25) to extrapolate the lattice Green's function from the imaginary axis. Typically, a few hundred Matsubara points are used in this extrapolation, with a frequency-dependent broadening. This procedure yields reliable results at low frequencies. Uncertainties arise mainly at higher frequencies and affect the position and width of the Hubbard bands.

The quasiparticle spectrum of $\mathrm{FeAsLaO}$ is seen to exhibit two main peaks, below and above $E_{F}$, separated by a narrow pseudogap slightly above $E_{F}$, and a lower Hubbard band below the $\mathrm{Fe} 3 d$ band region. The bare density of states also exhibits 


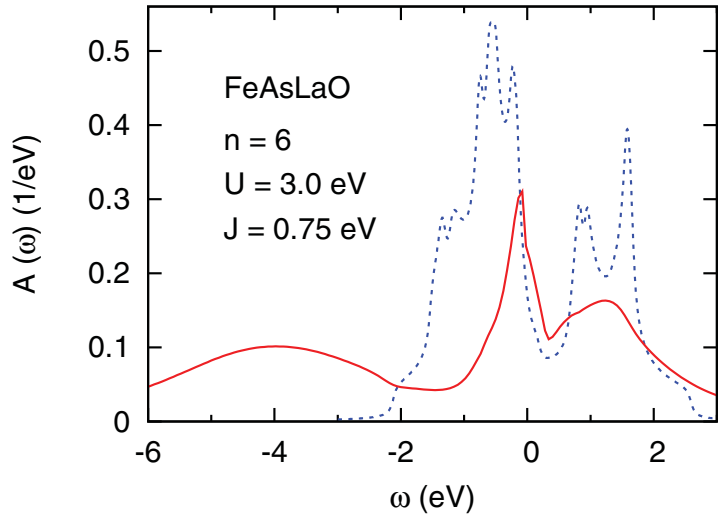

FIG. 1. (Color online) Fe $3 d$ quasiparticle spectra of FeAsLaO, calculated within ED DMFT $\left(n_{s}=15\right)$ for $U=3 \mathrm{eV}, J=0.75 \mathrm{eV}$ (solid red curve). The bare $3 d$ density of states is indicated by the dashed blue curve (Ref. 23).

pronounced peaks below and above $E_{F}$, and a deep minimum at $\sim 0.3 \mathrm{eV}$ above the Fermi energy. Comparing these spectra, one is tempted to conclude that Coulomb interactions lead to a significant narrowing of the occupied $3 d$ bands and to a strong shift toward $E_{F}$. Only a small fraction of the occupied spectral weight $(\sim 0.15 / 0.6$ per spin band) remains in the coherent peak near $E_{F}$, while the main part $(\sim 0.45 / 0.6)$ is distributed over the wide incoherent region. In the unoccupied region, the effect of Coulomb interactions is less severe. The main density-ofstates peak is merely broadened and the minimum at $\sim 0.3 \mathrm{eV}$ above $E_{F}$ is approximately preserved. Similar quasiparticle spectra have been obtained for a variety of pnictides. ${ }^{6,13-21,26,27}$ In principle, integer occupancy $n=6$ can also sustain a Mott phase. This phase occurs, however, at much larger Coulomb energies. $^{4,16,18}$

Although the scenario discussed above appears plausible, it is nontrivial to disentangle genuine many-body features from the complex single-particle aspects of the density of states. In order to identify interaction effects due to $U$ and $J$, we have carried out ED DMFT calculations for a Hamiltonian consisting of five identical subbands with a semielliptical density of states, where the width $W=4 \mathrm{eV}$ corresponds to typical pnictide compounds. Figure 2 shows spectral distributions for a wide range of Coulomb and exchange energies, $U=3, \ldots, 5 \mathrm{eV}$ and $J=0.5, \ldots, 1.0 \mathrm{eV}$. Most previous DMFT studies of pnictides used $U$ and $J$ values within this range. Spectra at $U=2 \mathrm{eV}$ for $J=0.5, \ldots, 0.9 \mathrm{eV}$ are qualitatively similar. For simplicity, the present calculations are performed using one bath level per $3 d$ orbital $\left(n_{s}=10\right)$. The results agree qualitatively with more accurate ones for two bath levels per orbital. Even though $U$ and $J$ span a wide range, the spectra are seen to be remarkably similar. In all cases, the interacting density of states exhibits peaks below and above $E_{F}$, separated by a pseudogap slightly above $E_{F}$. The peak-dip structure near $E_{F}$ is reminiscent of the one in the quasiparticle spectrum shown in Fig. 1. Since in the present model the bare density of states is featureless, the pseudogap is entirely due to the frequency-dependent self-energy. Moreover, the pseudogap depends only weakly on the values of $U$ and $J$ within the range quoted above, but it disappears at small $J$, so that only a broad quasiparticle peak remains. An example

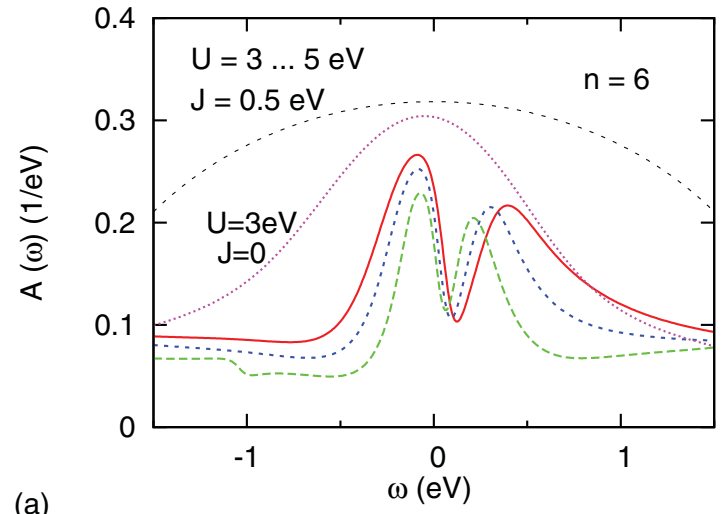

(a)

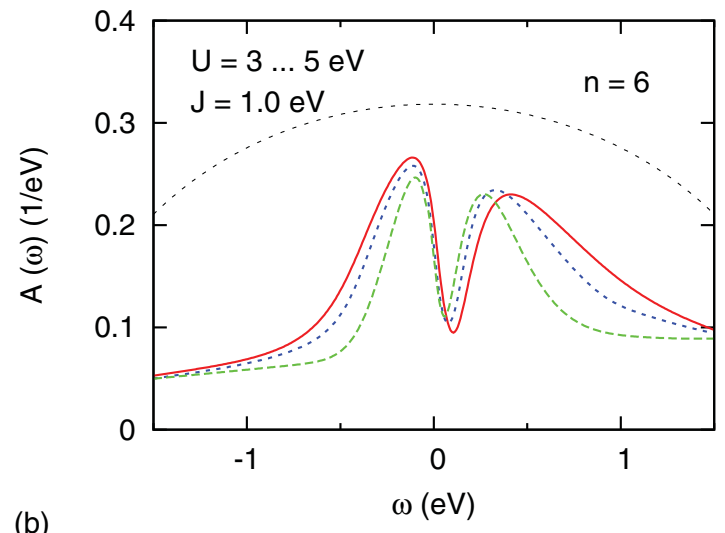

FIG. 2. (Color online) Low-energy region of spectral distribution of a degenerate five-band model at occupancy $n=6$, calculated within $\operatorname{ED~DMFT~}\left(n_{s}=10\right)$ for several Coulomb energies: $U=3 \mathrm{eV}$ (solid red curve), $U=4 \mathrm{eV}$ (short-dashed blue curve), and $U=5 \mathrm{eV}$ (long-dashed green curve). (a) $J=0.5 \mathrm{eV}$, (b) $J=1.0 \mathrm{eV}$. The spectra at intermediate values of $J$ are similar. The upper panel also shows the results for $U=3 \mathrm{eV}, J=0$ (dotted magenta curve). The bare density of states is indicated by the black dashed curve.

is shown in Fig. 2(a) for $U=3 \mathrm{eV}, J=0$. These results suggest that the pseudogap above $E_{F}$ is a generic feature caused by multiband Coulomb correlations within the $3 d$ shell, and that its existence depends crucially on realistic values of Hund coupling. The paramagnetic quasiparticle distribution of actual pnictides should therefore consist of a combination of correlation features associated with $J$ and signatures related to the bare density of states.

To illustrate the effect of Coulomb correlations on the self-energy, we show in Fig. 3 the imaginary part of $\Sigma\left(i \omega_{n}\right)$ for several occupancies. For $n \geqslant 6.2, \operatorname{Im} \Sigma\left(i \omega_{n}\right) \sim \omega_{n}$ at low energies, so that the system is a correlated Fermi liquid with a doping-dependent effective mass enhancement $m^{*} / m \approx$ $3, \ldots, 6$. At smaller occupancy, $\operatorname{Im} \Sigma$ reveals a nonzero onset, leading to bad-metallic behavior. As shown previously, ${ }^{19}$ the spin correlation function then changes from Pauli to Curie-Weiss behavior, indicating a spin freezing transition, in close analogy to the one found in the degenerate three-band model near $n=2 .{ }^{28}$ In addition, $\operatorname{Im} \Sigma\left(i \omega_{n}\right)$ exhibits a kink near $\omega_{n} \approx 0.1, \ldots, 0.2 \mathrm{eV}$, which is weak in the Fermi-liquid region, but becomes more intense at lower occupancy. As shown in Fig. 3(b), this kink is related to a resonance in 


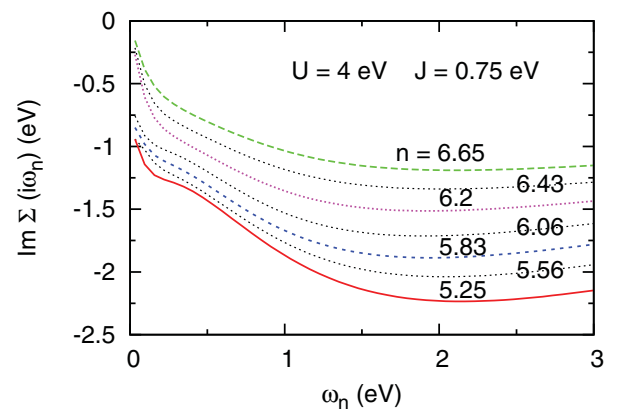

(a)
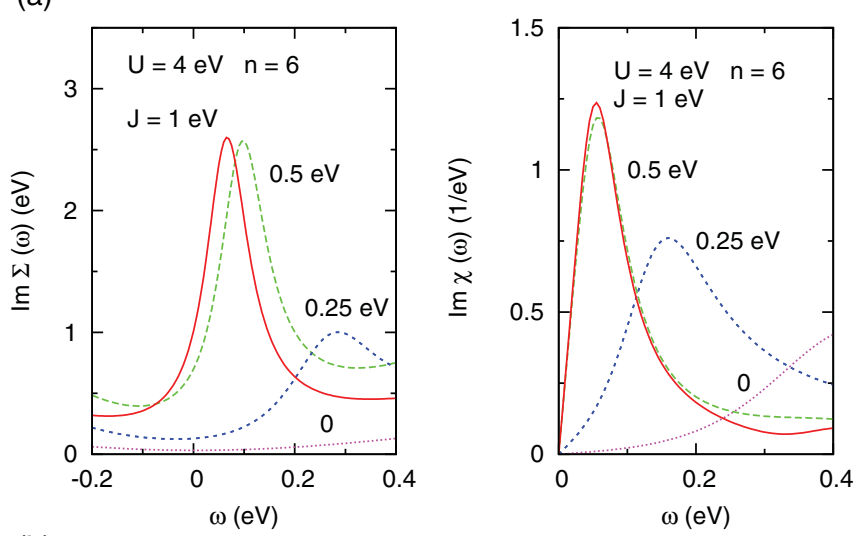

(b)

FIG. 3. (Color online) (a) Imaginary part of self-energy for a degenerate five-band model, calculated within ED DMFT $\left(n_{s}=15\right)$ as a function of Matsubara frequency for various occupancies at $U=4 \mathrm{eV}, J=0.75 \mathrm{eV}$. (b) Variation of self-energy (left) and spin susceptibility (right) with Hund coupling for $U=4 \mathrm{eV}, n=6$ $\left(n_{s}=10\right)$.

$\operatorname{Im} \Sigma(\omega)$, which is the origin of the pseudogap above $E_{F}$ in the spectra shown in Fig. 2. Remarkably, the resonance disappears at small $J$. For $J=0.5, \ldots, 1.0 \mathrm{eV}$, the local spin correlation function reveals a maximum below $0.1 \mathrm{eV}$, suggesting that the resonance in $\operatorname{Im} \Sigma(\omega)$ corresponds to a collective mode induced by spin fluctuations associated with Hund coupling. Thus, states in the resonance region have a greatly reduced lifetime. According to Kramers-Kronig relations, $\operatorname{Re} \Sigma(\omega)$ exhibits a positive slope near the resonance, so that some spectral weight is removed from the pseudogap region. Beyond this region, the slope of $\operatorname{Re} \Sigma(\omega)$ becomes negative again, giving rise to a kink in the dispersion of energy bands. ${ }^{29}$

An alternative, complementary understanding of the pseudogap can be achieved by realizing that the system at $n=6$ occupancy exists in proximity to the $n=5$ Mott insulator. As shown in Fig. 4, in the limit of half filling, the degenerate five-band model exhibits a Mott transition at a critical Coulomb energy that depends very sensitively on $J$, but is rather insensitive to the value of $U$. In particular, $U_{c}$ diminishes rapidly when $J$ increases: $U_{c} \approx 3 \mathrm{eV}$ for $J=0.25 \mathrm{eV}$ and $U_{c} \approx 1 \mathrm{eV}$ for $J=0.5 \mathrm{eV}$. (For $J=U / 4$ and $J=U / 6$, we find $U_{c} \approx 1.5 \mathrm{eV}$ and $U_{c} \approx 2.0 \mathrm{eV}$, respectively, in agreement with Ref. 4.) In contrast, because of the high orbital degeneracy, $J=0$ yields a Mott gap only at very large Coulomb energy $\left(U_{c} \approx 15 \mathrm{eV}\right)$. Thus, for $U$ and $J$ values usually employed in DMFT studies of iron pnictides (see the green/gray box), the half-filled system is a Mott insulator composed of localized $S=5 / 2$ spins. Upon

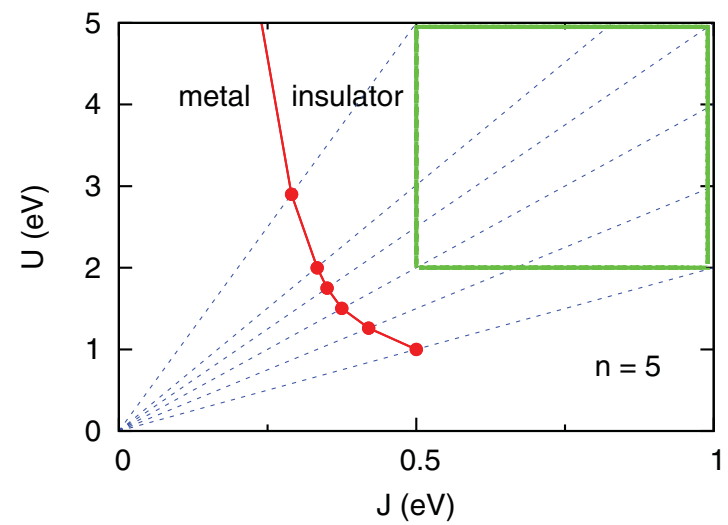

FIG. 4. (Color online) Paramagnetic phase diagram of degenerate five-band model at half filling. The dashed blue lines denote fixed ratios $J / U$ between $1 / 10$ and $1 / 2$. The dots represent the critical Coulomb energies of the Mott transitions along these lines. To the right (left) of the solid red curve, the system is insulating (metallic). The green/gray box indicates the range of $U$ and $J$ values commonly used in DMFT studies of iron pnictides. For $n=6$ the Mott phase occurs at much larger values of $U$ (Ref. 4), so that, approximately to the right of the solid red curve, the system is bad metallic.

electron doping, some of the localized states become itinerant, so that the density of states exhibits a narrow quasiparticle peak below $E_{F}$, and a concomitant minimum or pseudogap above $E_{F}$, in agreement with the spectra shown in Fig. 2. DMFT calculations as a function of doping (not shown here) reveal that, away from half filling, the upper Hubbard band quickly gets weaker and merges with the spectral feature above $E_{F}$, while the lower Hubbard peak remains rather stable. On the other hand, according to the phase diagram shown in Fig. 4 , in the absence of exchange interactions, the high orbital degeneracy yields a weakly correlated metal since $U \ll U_{c}$. This also holds at finite electron doping, so that the spectral distribution exhibits a broad quasiparticle peak, as indicated by the results for $U=3 \mathrm{eV}, J=0$ in Fig. 2(a). It is evident, therefore, that the collective mode in the self-energy shown in Fig. 3 and the associated pseudogap in the density of states (Fig. 2) obtained at realistic values of $J$ are a consequence of Hund coupling.

As pointed out above, the complex geometry and band structure of pnictides gives rise to strong crystal-field effects, causing appreciable orbital dependence of the self-energy and interacting density of states, as has been found in various DMFT studies. ${ }^{6,12-18}$ In addition, screening processes differ greatly among pnictide compounds, leading to substantial variation in Coulomb and exchange energies. ${ }^{23}$ Resonances in $\operatorname{Im} \Sigma(\omega)$ and the corresponding pseudogaps in the interacting density of states might appear therefore only in certain $3 d$ orbitals. This seems to be the case, as indicated, for instance, by the kinks found in some self-energy components of FeAsLaO. ${ }^{16}$

We also note that there exists at present appreciable uncertainty concerning the experimentally determined values of $U$ and $J$. Whereas recent $x$-ray absorption (XAS) and resonant inelastic $\mathrm{X}$-ray scattering (RIXS) on pnictides such as $\mathrm{BaFe}_{2} \mathrm{As}_{2}$ 
reveal $U \leqslant 2 \mathrm{eV}$ and $J \approx 0.8 \mathrm{eV},{ }^{30}$ other XAS measurements suggest $U \approx 3, \ldots, 4 \mathrm{eV}$ and $J \approx 0.8 \mathrm{eV} .{ }^{31}$ Moreover, while DMFT studies typically employ $U \approx 3, \ldots, 5 \mathrm{eV}$ and $J \approx$ $0.6, \ldots, 0.9 \mathrm{eV},,^{2,6,12-21}$ a recent mean-field analysis of neutron scattering and photoemission data yields $U \approx 1.5 \mathrm{eV}$ and $J \approx$ $0.3, \ldots, 0.6 \mathrm{eV} .^{32}$ Nonetheless, despite these experimental and theoretical uncertainties, the robustness of the pseudogap over a wide range of $U$ and $J$ (see Fig. 2), and the persistence of the Mott phase at half filling down to remarklably low Coulomb energies ( $U \approx 1 \mathrm{eV}$ for $J>0.5 \mathrm{eV}$, see Fig. 4), suggests that the characteristic peak-dip structure near $E_{F}$ seen in quasiparticle spectra of pnictides ${ }^{6,13-21,26}$ might be partially due to Coulomb correlations. Optical spectra in the paramagnetic phase then should exhibit a depletion of spectral weight close to $E_{F}$, as was recently predicted theoretically ${ }^{6}$ and observed experimentally in $\mathrm{BaFe}_{2} \mathrm{Se}_{2} \cdot{ }^{7-9}$ In the future, it would be very interesting to search more systematically for possible links between pseudogaps and collective modes in the self-energy of pnictides, and to study their variation with doping and temperature.

The picture presented in this Rapid Communication has more general validity beyond the present five-band model. Thus, pseudogaps generated by self-energy resonances due to Hund coupling are also found in the analogous three-band system. ${ }^{33}$ The main difference is that, because of the lower degeneracy, the boundary in Fig. 4 between metallic and insulating phases at half filling is shifted to larger $J$ (except at large $U)^{34,35}$ and that at $n=3 \pm 1$ the system is relatively farther from the Mott phase than at $n=5 \pm 1$.

Conclusion. In summary, Hund coupling in ferropnictides plays an important role in the formation of a high-energy pseudogap. To disentangle this gap from density-of-states effects, the noninteracting part of the Hamiltonian is simplified in terms of five degenerate semielliptical subbands. The pseudogap can then be identified as a many-body feature that is stable over a wide range of Coulomb and exchange energies, and that is related to a collective mode in the self-energy associated with spin fluctuations. The nature of this mode can also be understood by viewing the system as a doped $n=5$ Mott insulator, where itinerant electrons coexist with localized spins. This picture is consistent with the observation that at finite doping the spectral distribution exhibits a narrow quasiparticle peak below $E_{F}$ and a concomitant minimum above $E_{F}$, while most of the spectral weight resides in the lower Hubbard band. The crucial role of Hund exchange in this scenario is evident from the fact that, due to the high orbital degeneracy, at small values of $J$ the pseudogap disappears and the system is a weakly correlated metal. Thus, the high-energy pseudogap is directly linked to the realistic magnitude of Hund's rule coupling.

I like to thank Hiroshi Ishida for fruitful discussions. The DMFT calculations were carried out on the Jülich Juropa machine.
${ }^{1}$ Y. Kamihara, T. Watanabe, M. Hirano, and H. Hosono, J. Am. Chem. Soc. 130, 3296 (2008).

${ }^{2}$ K. Haule and G. Kotliar, New. J. Phys. 11, 025021 (2009).

${ }^{3}$ M. D. Johannes and I. I. Mazin, Phys. Rev. B 79, 220510(R) (2009).

${ }^{4}$ A. M. Läuchli and Ph. Werner, Phys. Rev. B 80, 235117 (2009).

${ }^{5}$ S. Zhou and Z. Wang, Phys. Rev. Lett. 105, 096401 (2010).

${ }^{6}$ Z. P. Yin, K. Haule, and G. Kotliar, Nat. Phys. 7, 294 (2011).

${ }^{7}$ W. Z. Hu, J. Dong, G. Li, Z. Li, P. Zheng, G. F. Chen, J. L. Luo, and N. L. Wang, Phys. Rev. Lett. 101, 257005 (2008).

${ }^{8}$ N. L. Wang, W. Z. Hu, Z. G. Chen, R. H. Yuan, G. Li, G. F. Chen, and T. Xiang, e-print arXiv:1105.3939.

${ }^{9}$ A. A. Schafgans, S. J. Moon, B. C. Pursley, A. D. LaForge, M. M. Qazilbash, A. S. Sefat, D. Mandrus, K. Haule, G. Kotliar, and D. N. Basov, e-print arXiv:1106.3114.

${ }^{10}$ M. M. Qazilbash, J. J. Hamlin, R. E. Baumbach, Lijun Zhang, D. J. Singh, M. B. Maple, and D. N. Basov, Nat. Phys. 5, 647 (2009).

${ }^{11}$ M. Xu, P. Richard, K. Nakayama, T. Kawahara, Y. Sekiba, T. Qian, M. Neupane, S. Souma, T. Sato, T. Takahashi, H.-Q. Luo, H.-H. Wen, G.-F. Chen, N.-L. Wang, Z. Wang, Z. Fang, X. Dai, and H. Ding, Nat. Commun. 2, 392 (2011).

${ }^{12}$ K. Haule, J. H. Shim, and G. Kotliar, Phys. Rev. Lett. 100, 226402 (2008).

${ }^{13}$ L. Craco, M. S. Laad, S. Leoni, and H. Rosner, Phys. Rev. B 78, 134511 (2008).

${ }^{14}$ M. Aichhorn, L. Pourovskii, V. Vildosola, M. Ferrero, O. Parcollet, T. Miyake, A. Georges, and S. Biermann, Phys. Rev. B 80, 085101 (2009).

${ }^{15}$ S. L. Skornyakov, A. V. Efremov, N. A. Skorikov, M. A. Korotin, Y. A. Izyumov, V. I. Anisimov, A. V. Kozhevnikov, and D. Vollhardt,
Phys. Rev. B 80, 092501 (2009); S. L. Skornyakov, N. A. Skorikov, A. V. Lukoyanov, A. O. Shorikov, and V. I. Anisimov, ibid. 81, 174522 (2010).

${ }^{16}$ H. Ishida and A. Liebsch, Phys. Rev. B 81, 054513 (2010).

${ }^{17}$ P. Hansmann, R. Arita, A. Toschi, S. Sakai, G. Sangiovanni, and K. Held, Phys. Rev. Lett. 104, 197002 (2010).

${ }^{18} \mathrm{M}$. Aichhorn, S. Biermann, T. Miyake, A. Georges, and M. Imada, Phys. Rev. B 82, 064504 (2010).

${ }^{19}$ A. Liebsch and H. Ishida, Phys. Rev. B 82, 155106 (2010).

${ }^{20}$ M. Aichhorn, L. Pourovskii, and A. Georges, Phys. Rev. B 84, 054529 (2011)

${ }^{21} \mathrm{Ph}$. Werner, M. Casula, T. Miyake, F. Aryasetiawan, A. J. Millis, and S. Biermann, e-print arXiv:1107.3128.

${ }^{22}$ A. Georges, G. Kotliar, W. Krauth, and M. J. Rozenberg, Rev. Mod. Phys. 68, 13 (1996).

${ }^{23}$ T. Miyake, K. Nakamura, R. Arita, and M. Imada, J. Phys. Soc. Jpn. 79, 044705 (2010)

${ }^{24}$ M. Caffarel and W. Krauth, Phys. Rev. Lett. 72, 1545 (1994).

${ }^{25}$ W. H. Press, B. P. Flannery, S. A. Teukolsky, and W. T. Vetterling, Numerical Recipes in Fortran 77 (Cambridge University Press, Cambridge, UK, 1986-1992), p. 106.

${ }^{26}$ H. Ikeda, R. Arita, and J. Kunes, Phys. Rev. B 82, 024508 (2010).

${ }^{27}$ Density-density exchange treatments amount to an enhancement of Coulomb interactions (Ref. 16) and can lead to dramatic redistribution of spectral weight near $E_{F}$ (Ref. 18).

${ }^{28} \mathrm{P}$. Werner, E. Gull, M. Troyer, and A. J. Millis, Phys. Rev. Lett. 101, 166405 (2008).

${ }^{29}$ For correlation-induced kinks in one-band models, and their relation to collective modes due to spin fluctuations, see the following: 
K. Byczuk, M. Kollar, K. Held, Y.-F. Yang, I. A. Nekrasov, T. Pruschke, and D. Vollhardt, Nat. Phys. 3, 168 (2007); C. Raas, P. Grete, and G. S. Uhrig, Phys. Rev. Lett. 102, 076406 (2009); P. Grete, S. Schmitt, C. Raas, F. B. Anders, and G. S. Uhrig, e-print arXiv:1107.1370.

${ }^{30}$ W. L. Yang, A. P. Sorini, C-C. Chen, B. Moritz, W.-S. Lee, F. Vernay, P. Olalde-Velasco, J. D. Denlinger, B. Delley, J.-H. Chu, J. G. Analytis, I. R. Fisher, Z. A. Ren, J. Yang, W. Lu, Z. X. Zhao, J. van den Brink, Z. Hussain, Z.-X. Shen, and T. P. Devereaux, Phys. Rev. B 80, 014508 (2009).
${ }^{31}$ V. I. Anisimov, E. Z. Kurmaev, A. Moewes, and I. A. Izyumov, Physica C 409, 442 (2009).

${ }^{32}$ Q. Luo, G. Martins, D.-X. Yao, M. Daghofer, R. Yu, A. Moreo, and E. Dagotto, Phys. Rev. B 82, 104508 (2010).

${ }^{33}$ A. Liebsch and H. Ishida, J. Phys. CM (accepted for publication as Topical Review).

${ }^{34} \mathrm{Ph}$. Werner, E. Gull, and A. J. Millis, Phys. Rev. B 79, 115119 (2009).

${ }^{35}$ L. de' Medici, Phys. Rev. B 83, 205112 (2011); L. de' Medici, J. Mravlje, and A. Georges, e-print arXiv:1106.0815. 